\title{
Analysis of Circulation of Neuronal Activity in the Waking Cortex ${ }^{1}$
}

\author{
P. RINALDI, G. JUHASZ ${ }^{2}$ AND M. VERZEANO
}

Department of Psychobiology, University of California, Irvine, CA 92717

(Received 5 February 1976)

\begin{abstract}
RINALDI, P., G. JUHASZ AND M. VERZEANO. Analy sis of circulation of neuronal activity in the waking cortex. BRAIN RES. BULL. 1(5) 429-435, 1976. - It is well known that the rhy thmic activity of the cerebral cortex is closely associated with a highly organized circulation of neuronal impulses through the networks in which the activity develops. The present study has attempted to determinc by quantitative methods, the consistency of this circulation and of its association with the cortical waves, over relatively long periods. Cortical gross waves and neuronal activity have been recorded by means of arrays of extracellular microelectrodes, in freely moving cats as well as in cats immobilized with flaxedil. Autocorrelations have been performed on trains of rhythmic waves and on associated clusters of action putentials; cross-correlations and nultiple correlations have been performed between waves and action potentials and between action potentials generated by different groups of neurons. It has been found that the rhythmicity of the waves, the rhythmicity of the associated clusters of action potentials, the time and phase relations between them, and the circulation of neuronal activity through the networks, remain highly consistent over periods as long as two hours.
\end{abstract}

Circulation of activity Neuronal activity Cerebral cortex

INVESTIGATIONS conducted during the last two decades have shown that the development of rhythmic brainwaves (synchronization), in the thalamus and in the cortex, is based on summations of excitatory and inhibitory postsynaptic potentials, maintained through complex neuronal interactions $[1,2,3,4,10,11,12,13]$. The mechanism on which these interactions are based involves a highly organized circulation of neuronal activity through thalamic and cortical networks, which has been detected and analyzed by means of recordings obtained, simultaneoulsy, with several microelectrodes $[1,23,25,26,27,29,30,32$, 33].

The successive passages of the circulating impulses through the networks are closely and consistently associated with the periodicity of the rhythmic waves. There is experimental evidence which suggests that this organized circulation of neuronal activity may play a role in several physiological processes: in the distribution of excitation and inhibition in the neuronal networks whose action is involved in the transition from wakefulness to sleep [23, 30,331 ; in the transport of sensory information from one region of the brain to another $[22,30]$; and in the processing and storage of sensory information $[5,22,24]$. It is, therefore, important to determine whether the circulation of activity goes on for periods sufficiently long to function as a fundamental mechanism in physiological processes whose implementation may require minutes or hours rather than seconds. Since the direction and the position of the pathway along which the circulation proceeds through the network and the phase relations between clusters of neuronal impulses and rhythmic waves or between the activities of different neurons may show some variability, the consistency of these relations is difficult to evaluate by ordinary measurements. For this reason, a series of investigations has been undertaken in which the techniques of autocorrelation and cross correlation have been applied to the study of the circulation of neuronal activity.

The results of the first phase of this study, which deals with the investigation of these processes in the waking cortex, are presented in this report.

\section{METHOD}

Multiple microelectrodes made of platinum-iridium wire, sharpened electrolytically to diameters of 0.5 to $2 \mu$ and insulated with glass [34] were stereotoxically implanted in the brain of cats, under barbiturate anesthesia. The animals were allowed to recover and, a few days to a few weeks later, the neuronal activity and the gross waves were studied in the freely moving animal, in the waking state, in the absence of any effects due to anesthetics or other drugs. In other experiments, the recordings were obtained from waking cats immobilized with flaxedil and maintained with artificial respiration. The technique for the simultaneous implantation of several microelectrodes was described elsewhere [24].

\footnotetext{
${ }^{1}$ Aided by Grant NS-07145 from the National Institution of Health.

${ }^{2}$ Visiting Investigator from the Institute of Psychology of the Hungarian National Academy of Sciences.
} 
The neuronal activity and the gross waves were recorded through amplifiers with an input impedance of over 200 megohms and a frequency response of $1 \mathrm{~Hz}$ to $10 \mathrm{KHz}$. The output of the amplifiers was recorded on magnetic tape and the data were analyzed subsequently. By using appropriate filter circuits it was possible to separate the gross waves from the neuronal action potentials. Microelectrodes of larger diameters detected the activity of several neurons and, accordingly, recorded action potentials of several amplitudes. These pulses of different amplitudes, representing the activity of several neurons or groups of neurons were separated by means of amplitude discriminators $[29,30]$.

The groups of neurons whose activities were correlated were determined in the following way: (a) the action potentials recorded by each microelectrode were separated by means of the amplitude discriminators into two ranges, one range containing the high amplitudes and another containing the low amplitudes, with a sufficient gap between them, to insure that the two ranges represented the activilies of two different groups of neurons of different size or different type $[7,15,19,24,31]$. Correlating these two ranges of action potentials provided the means for studying the relations between the activities of large and small neurons within the territory surveyed by one microelectrode; (b) the distance between the tips of the microelectrodes was large enough to insure that each microelectrode surveyed a different territory. Correlating the action potentials recorded by different microelectrodes provided the means for studying the relations between the activities of groups of neurons located in neighboring but different territories. Thus, one array of three microelectrodes could clearly and unequivocally detect the activities of at least six groups of neurons: three groups generating action potentials of high amplitudes and three groups generating action potentials of low amplitudes (Fig. 1). The activity of single neurons was detected either by selecting, through the discriminators, the action potentials whose constant amplitude was markedly and consistently higher than all other amplitudes present in the record, or by using microelectrodes of small diameters which would detect the activity of isolated neurons only. The gross waves were recorded either by one of the microelectrodes in the array or by a gross electrode $(0.2 \mathrm{~mm}$ in dia.) located $0.5 \mathrm{~mm}$ from the center of the array.

Autocorrelations were performed on waves and trains of action potentials in order to determine the consistency of their periodicity; cross correlations were performed between gross waves and trains of action potentials or between trains of action potentials generated by different neurons or groups of neurons in order to determine the consistency of their time and phase relations. Correlations of action potentials were made by two methods. With one method, the trains of pulses provided by the amplitude discriminator and representing trains and clusters of action potentials produced by groups of neurons, were translated. by means of integrators, into waves whose amplitude was proportional to the frequency of neuronal discharge (Fig. $2)$. By autocorrelating or cross correlating these waves it was possible to study the periodicity of clusters of action potentials generated by one group of neurons or the time and phase relations between clusters of action potentials

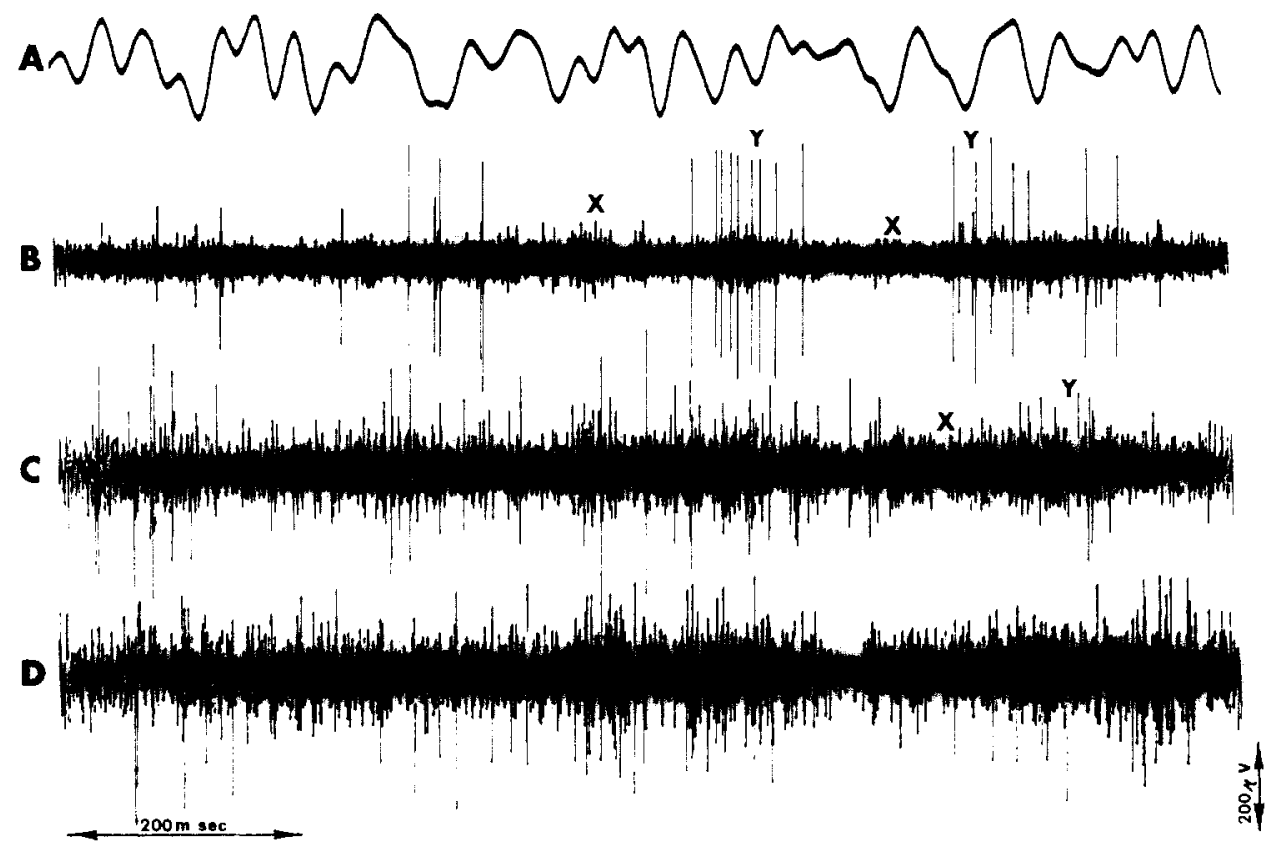

FIG. 1. Gross wave (A) and neuronal activity (B, C, D) in the primary visual cortex of the cat, recorded by means of an array of three microelectrodes displayed medio-laterally along a straight line. The microelectrodes are at different depths within the cortex: the medial (B) at $270 \mu$, the middle (C) at $550 \mu$, and the lateral (D) at $750 \mu$. Distances between the tips are: B to C $370 \mu$; C to D $310 \mu$. Action potentials of markedly different amplitude, such as $X$ and $Y$, represent activities of neurons of different size. Note the alternation, in time, of action potentials of low and high amplitudes (at $X$ and Y). Negative down. Animal under Flaxedil. 

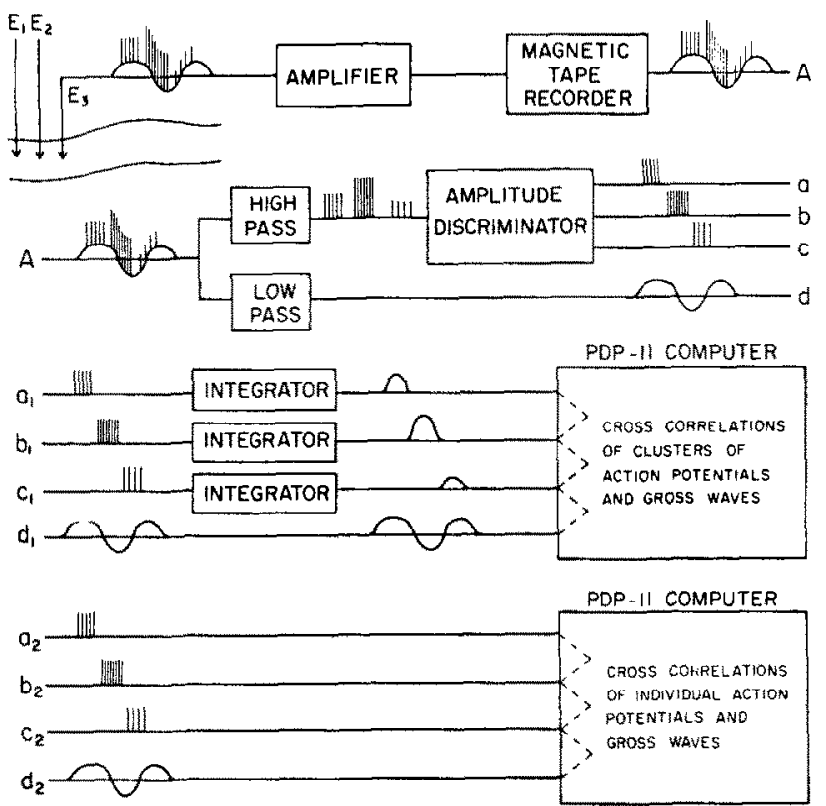

FIG. 2. Arrangement and operation of electronic devices and computers. The sequence of operations is shown for one microelectrode only $\left(\mathrm{E}_{3}\right)$. The output of the amplifier, containing action potentials and gross waves is recorded on one channel of the magnetic tape recorder. During subsequent play-back, action potentials and gross waves are separated by appropriate filters. The gross waves are fed, directly into the analog to digital converter of the PDP-11 computer. Action potentials of different amplitudes, representing the activities of different neurons or groups of neurons, are separated by an amplitude discriminator in such a way that each amplitude appears, on a different channel, in the form of a sequence of identical puises. By means of integration these sequences of pulses $\left(a_{1} ; b_{2} ; c_{1}\right)$ are transcribed into waves whose amplitude is proportional to the frequency of the pulses and, therefore, to the frequency of discharge of the different neurons or groups of neurons. These waves are fed into the analog to digital converter of the PDP-11. This mode of analysis is used for clusters of action potentials (such as $X$ and $Y$ in Fig. 1). Individual action potentials are taken from the output of the amplitude discriminators directly to the analog to digital converter of the PDP-11 $\left(a_{2} ; b_{2} ; c_{2}\right)$.

generated by different groups of neurons; by cross correlating them with the gross waves it was possible to study the time and phase relations between clusters of action potentials and gross waves. With the other method, the pulses provided by the amplitude discriminator were correlated directly, without integration, by using an averaging process, similar to that which is used in computing post-stimulus histograms; however, instead of a stimulus pulse, the computer was triggered by an action potential (Fig. 2). Thus, should a correlation exist between two trains of action potentials, triggering with the action potentials of one train and averaging the other with respect to time, a high probability of occurrence should appear at the time at which the action potentials of the second train follow those of the first train. By reversing the process and triggering with the action potentials of the second train a correlation might be found which would show that the action potentials of the first train follow those of the second train. The great advantage of this method, in addition to its simplicity, is that correlations are not limited to two channels containing one train of action potentials each; they can be extended to any number of channels that the computer can handle, by triggering with the action potentials of one train and averaging all the others in time, on as many channels as needed (Figs. 2 and 6). The method can also be applied to correlations between action potentials and gross waves, by triggering with the former and averaging the latter.

All computations were made by means of a DEC PDP-11 computer. The location of the points from which recordings were obtained was determined by serial section of the brain and histological preparation.

\section{RESULTS}

General Consistency and Continuity of the Circulation of Neuronal Activity

Recordings obtained at intervals as long as two hours show that the rhythmicity of the gross waves, the rhythmicity of the neuronal discharge, and the time and phase relations between them remain consistent over long periods. Similarly a high degree of consistency is found in the time and phase relations between the neuronal discharge of several groups of neurons located within the networks simultaneously surveyed by three microelectrodes. This is illustrated in Fig. 3 which shows a series of autocorrelations and cross correlations performed on recordings obtained from the primary visual cortex of one animal, first at the beginning of the experimental session (A) and two hours later (B), without any changes in the position of the microelectrodes during the intervening time. It can be seen that the rhythmicity of the waves (Al and B1), the rhythmicity of the neuronal discharge ( $A 2$ and $B 2$ ), the time and phase relations between the two (A3 and B3), as well as the relations between the discharge of two groups of neurons recorded by different microelectrodes (A4 and B4) are the same at the beginning of the experiment and two hours later.

\section{Time and Phase Relations Between the Activities of} Different Groups of Neurons

When the activities of two groups of neurons recorded by the same microelectrode are cross correlated (action potentials of high amplitude against action potentials of low amplitude), it is found that they are out of phase, in most cases by 180 degrees. This is illustrated in Fig. 4A1 which shows the results of a cross correlation performed on the clusters of action potentials of high amplitude and low amplitude, recorded by one microelectrode. It can be seen that maximum negative correlation occurs very close to time zero and maximum positive correlation occurs at 160 msec to the right and $160 \mathrm{msec}$ to the left of time zero, which indicates that the clusters of action potentials of high amplitude alternate with the clusters of action potentials of low amplitude at intervals of $160 \mathrm{msec}$. When such recordings are obtained, simultaneously, with three microelectrodes whose tips are displayed along a straight line in the medio-lateral direction, seperated by distances of 300 to $380 \mu$, it is found that similar time and phase relations between clusters of action potentials of high and low amplitudes occur in the territory immediately surrounding the tips of each one of the microelectrodes (Fig. 4A at 1,2, 3 ). By contrast the clusters of action potentials of high amplitude recorded by one microelectrode, occur with the 
A

B
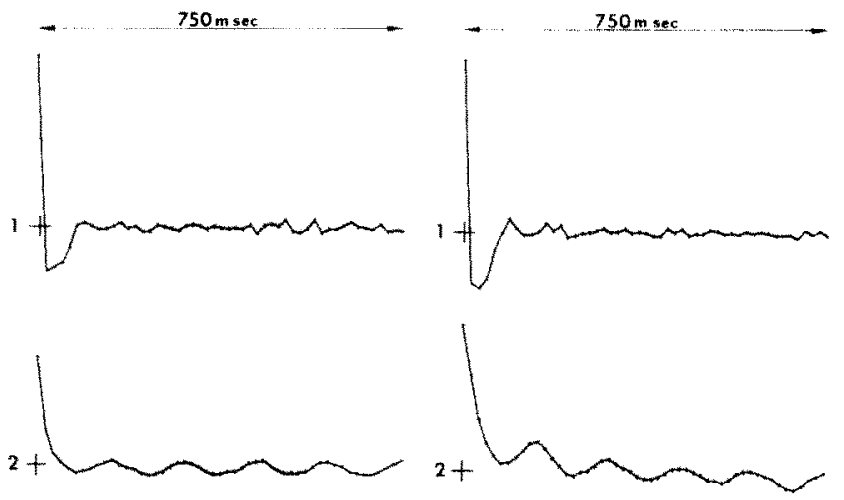

3 wherentymarm

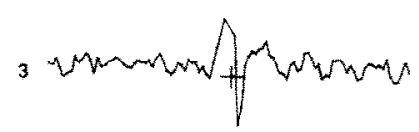
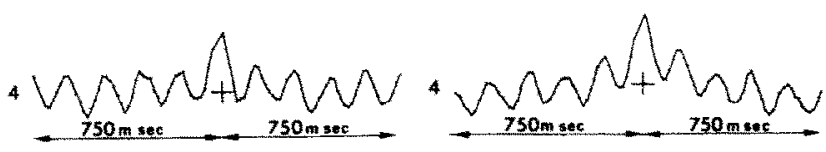

FIG. 3. Consistency of periodicity and of time and phase relations in the activity of a neuronal network in the visual cortex of the cat, as determined by auto and cross correlation studies. Computations performed on recordings obtained at the beginning of the experiment (Column A) and $2 \mathrm{hr}$ later (Column B). (1) Autocorrelation of gross waves; (2) Autocorrelation of sequences of clusters of action potentials of $140 \mu \mathrm{v}$ amplitude or higher, recorded by the medial microelectrode of an array of three; (3) Cross correlation between the same sequence of clusters of action potentials and the gross waves recorded with the same mictoelectrode; (4) Cross correlation between the same sequence of clusters of action potentials and a similar sequence (110 $\mu \mathrm{v}$ or higher) recorded by a neighboring microelectrode. Computations were performed on $2 \mathrm{~min}$ of recording in Column A. on $5 \mathrm{~min}$ in Column B. Arrangement of microelectrodes same as in Fig. 1. Animal under Flaxedil.

same hythmicity as the cluster of action potentials of high amplitude recorded by the other two microelectrodes in the array, and are fully in phase with them (Fig. 4B 1 and 2). Occasionally similar time and phase relations are found between clusters of action potentials of high amplitude generated by single neurons, recorded by two microelectrode whose tips are separated by a distance of $380 \mu$ (Fig. 4B at 3 ).

A high degree of consistency in the periodicity of the neuronal activity, as well as in the relations between the activities of different groups of neurons, is found in other regions of the cortex. This is illustrated in Fig. 5 which shows a series of cross correlations performed on recordings obtained with two micorelectrodes from the association cortex of an unrestrained, unanesthetized cat. The cross correlation between the action potentials of high amplitudes recorded by each of the two microelectrodes is out of phase, the clusters of action potentials recorded by the anterior microelectrode lagging the clusters of action potentials recorded by the posterior microelectrode by about $33 \mathrm{msec}$ (A3); the cross correlation between clusters of large and clusters of small action potentials are 180 degrees out of phase, in each one of the territories surveyed by the two microelectrodes (B1 and B2).
A

B
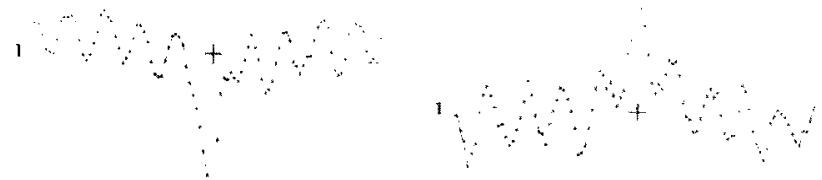

$2 \because \because y+b y+3$

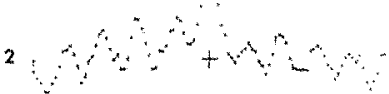

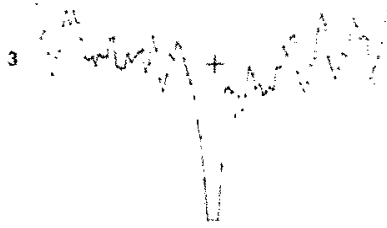

$3 \therefore \therefore \theta$

$-750 \mathrm{msec}$

1IG. 4. Time and phase relations between the activities of different neurons and groups of neurons recorded, by an array of three microelectrodes, from the visual cortex of the cat. Column A: cross correlations between clusters of high amplitude and low amplitude action potentials recorded by the medial (1), the middle (2) and the lateral (3) microelectrodes of the array. Note that high amplitude and low amplitude action potentials are $180^{\circ}$ out of phase. Column $B$ : cross correlations between clusters of action potentials of high amplitudes recorded by the medial and the middle microclectrodes (1); by the middle and the lateral microelectrodes (2); and between single cells recorded by the medial and middle microelectrodes ( 3 ) of the array. Note that they arc fully in phase. Microelectrodes arrangement and distances, as in Fig. 1 and 3. Animal under Flaxedil.

\section{Time and Phase Relations Between Neuronal Activity and Gross Waves}

The sequences of gross waves show a very consistent periodicity. The degree of periodicity is more marked when the gross waves are recorded with a gross electrode located close to the center of the array than when they are recorded with the tip of one of the microelectrodes. Figure 5 , column A at 1 shows this consistent periodicity as recorded from a gross electrode in the association cortex.

Similarly, consistent time and phase relations are found between the gross waves and the clusters of action potentials recorded by the microelectrodes. This is illustrated in Fig. 3 and 5 which show these relations as they occur in the visual cortex and in the association cortex, respectively. In Fig. 3 (at A3) it can be seen that there is correlation between the gross waves and the clusters of action potentials of high amplitude recorded by the same microelectrode, with the waves lagging the action potentials by about $33 \mathrm{msec}$. In the same figure (at B3) it can be seen that the relation between gross waves and action potentials has remained the same two hours later. In Fig. 5 (at A2) it can be seen that there is correlation between the waves recorded by the gross electrode and the clusters of action potentials of high amplitude recorded with a microelectrode, the wave leading the action potentials by about 65 msec.

Recordings Obtained With Permanently Implanted Microelectrodes

Recordings obtained from the association cortex in 
A

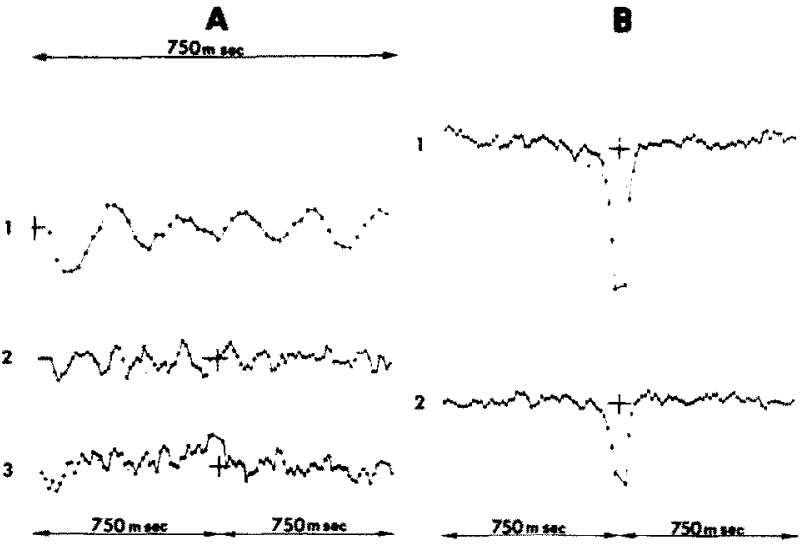

FIG. 5. Time and phase relations between the activities of groups of neurons and gross waves recorded by an array composed of two microelectrodes and a gross electrode, in the association cortex of the cat. Column A: autocorrelation of gross waves recorded by gross electrode (1); cross correlation between gross wave recorded by gross electrode and clusters of action potentials of high amplitude recorded by the posterior microelectrode (2); cross correlation between clusters of action potentials of high amplitude recorded by each of the two microelectrodes (3). Column B: cross correlation between clusters of action potentials of high and low amplitude recorded by posterior microelectrode (1) and by anterior microelectrode (2). Note that in $A(2)$ the waves lead the action potentials by $65 \mathrm{msec}$; in $A(3)$ the action potentials recorded by the posterior microelectrode lag behind the ones recorded by the anterior microelectrode by $33 \mathrm{msec}$; and in $B(1$ and 2$)$ the action potentials of high and low amplitude recorded by either microelectrode are $180^{\circ}$ out of phase. Electrode arrangement: tip of gross electrode and tips of microelectrodes formed a right triangle; the gross electrode and one microelectrode were displayed along an anteroposterior line, the microelectrode being posterior and below at a distance of $570 \mu$; the other microelectrode was located laterally and below at a distance of $535 \mu$ from the gross electrode. Unrestrained animal with implanted electrodes.

unanesthetized, unrestrained animals show the same consistency in the time and phase relations between the action potentials generated by the different groups of neurons and between neuronal activity and gross waves, as do the recordings obtained from the primary visual cortex in animals maintained in the stereotaxic instrument, under flaxadil (Fig. 5).

\section{Correlations of Individual Action Potentials Without Integ- ration}

The higher resolutions provided by this method permit the detection of interactions which occur very rapidly. This is illustrated in Fig. $6 \mathrm{~A}$ and $\mathrm{B}$. Both sections show correlations between several trains of action potentials recorded by the same array of microelectrodes in the same experiment as that illustrated in Fig. 3 and 4. In Fig. 6A the computer is triggered by the small action potentials $(30-50$ 4) recorded by the medial microelectrode, and several trains of individual action potentials, recorded by the three microelectrodes in the array, are simultaneously averaged in time, on a fast sweep. It can be seen that different groups of neurons discharge in a definite and very rapid sequence (at 1, 2, 3, $x-y$, and at 5,6,7). In Fig. 6B the same computation is performed on a slower time base with the gross wave being averaged simultaneously. In this case, too,
1

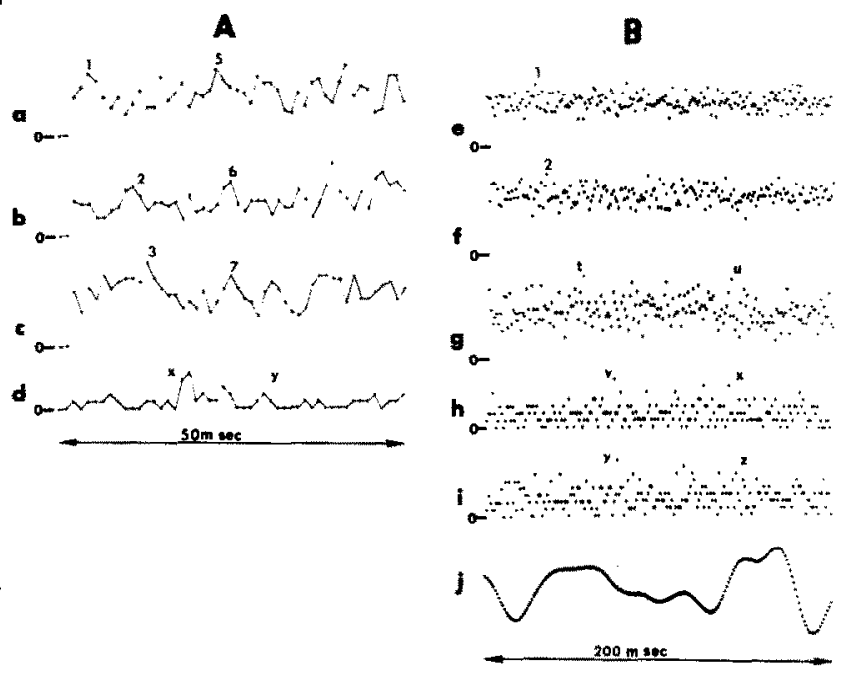

FIG. 6. Correlations of individual action potentials (without integration) recorded by an array of three microelectrodes from the visual cortex of the cat. Column A: computer is triggered by low amplitude $(30-50 \mu \mathrm{v})$ action potentials recorded by medial microelectrode; maximum probability of discharge occurs, sequentially, for low amplitude action potentials recorded by medial (a), middle (b) and lateral (c) microelectrodes, at 1,2,3; maximum probability of occurrence of high amplitude action potentials recorded by medial microelectrode follows at $x-y$ (d); another sequence of high probabilities of discharge of low amplitude action potentials follows at $5,6,7$. Column $B$ : computer is triggered as in $A$; maximum probabilities of discharge occur, sequentially, for low amplitude action potentials recorded by medial and lateral microelectrodes ( $e$ and $f$, and for high amplitude action potentials recorded by the lateral (g), medial (h) and middle (i) microelectrodes, at $1,2, t-u, v-x$ and $y-z$.

there is a definite sequence in the discharge of the different groups of neurons (at $1,2, t-u, v-x$, and $y-z$ ) as well as a clear correlation between this discharge and the gross wave.

\section{DISCUSSION}

The most remarkable finding in this series of experiments is the consistency in the time and phase relations between the discharge of the groups of neurons which are implicated in the circulation of activity associated with cortical rhythmicity. It is well known that when the activity of the cortex is highly rhythmic, the discharge of many of the cortical neurons involved in the pathway of circulation is markedly clustered $[23,25,28,30,32,33]$. By applying the correlation studies to these clusters of action potentials, as well as to individual action potentials, recorded over long periods, it has been possible to ascertain that the circulation of activity is not a fleeting phenomenon which occurs occasionally, or accidentially, but a highly stable process resulting from the organized interactions of many groups of neurons.

At the beginning of the development of techniques for multineuronal recording, it was believed that differences in the amplitude of the action potentials were due to different distances between the active neurons and the tip of the recording microelectrode $[23,28,32]$. In recent years it was found that, within the volume of neural tissue surveyed by the tip of a metallic microelectrode, which can be visualized as a sphere with a diameter of 100 to $200 \mu$, large 
differences in the amplitude of the action potentials are related to the size of the active neurons rather than to their distance from the recording point $[7,15,24,31]$

Furthermore, by recording the activity of the cat's motor cortex, while stimulating, antidromically, the fibers of the pyramidal tract, Tyler et al. [19] have demonstrated that large action potentials represent the activity of pyramidal cells while small action potentials represent the activity of interneurons. In the investigations reported here the analysis of the data focused on two sizes of action potentials: the very large $(80-400 \mu)$ and the very small $(30-50 \mu)$. The gap between the two sizes insured that these two groups of action potentials were generated by very different neurons, most probably pyramidal cells and interneurons. Since the activity of the cortical network was recorded with two or three microelectrodes simultaneously, and since each microelectrode in the array provided information on one group of large and one group of small neurons, the relations between the activities of four to six such groups could be studied, with a clear distinction between the large neurons which were, most probably, pyramidal cells, and the small neurons. most probably interneurons.

It appears from this study that several groups of neurons discharge in specific sequences, with specific time and phase relations between small and large neurons: (1) a very rapid sequence involving three groups of small neurons discharging about $2.5 \mathrm{msec}$ apart (Fig. 6A, at 1, 2, 3) followed, 2.5 msec later, by a burst discharge of large neurons (at $x-y)$; as soon as this burst discharge begins, a second sequence of rapid discharge of the small neurons may occur (at $5,6,7) ;(2)$ a slow sequence involving two groups of small neurons, discharging about $20-40 \mathrm{msec}$. apart (Fig. $6 \mathrm{~B}$, at 1,2$)$ followed by burst discharges of three groups of large neurons (at $t-u: v-x$; and $y-z)$, and related to a gross wave of about $160 \mathrm{msec}$ period (Fig. $6 \mathrm{~B}$ at j); and (3) an alternation, at 130 to $160 \mathrm{msec}$ intervals of the discharge of large and small cells (Fig, 1 at $x-y$ and Fig, 4A and 5B).

On the basis of these findings, and assuming that the small cells are interneurons and the large ones are pyramidal cells, it may be suggested that the three sequences of neuronal discharge may represent three different types of circuits involved in the development of cortical rhythmicity: (1) a short cortical feedback loop, containing only a few synaptic junctions and a few interneurons and pyramidal cells, based, possibly on recurrent inhibitory pathways and on dendro-dendritic action $\{18\}$ and functioning within a larger cortical region; (2) a longer cortical feedback loop, containing a larger number of synaptic junctions and of neurons and functioning within a small cortical region; and (3) a cortico-thalamic feedback loop, containing a very large and complex network of neurons and synaptic junctions, functioning within and between a large number of cortical regions and one or several thalamic nuclei, and leading to the development of the alternating discharge of small and large cortical cells. Strong evidence supporting this possibility is provided by the occurrence of identical alternations of small and large cell discharge in the thalamic nuclei, in close relation to the development of thalamic and cortical rhythmicity $[29,30]$ and by recent anatomical and physiological data $[6,8,9$. $16,17,20,211$ clearly indicating the existence and operation of cortico-thalamic feed back loops.

Thus, the findings provided by this series of investigam tions indicate not only that the organized circulation of activity through cortical networks is a continuous and consistent process closely related to cortical rhythmicity, but that it follows along feedback loops which are highly complex but may, nevertheless, be effectively studied by the methods of single-neuronal, multi-neuronal and multiple microelectrode recordings, combined with autocorrelation, cross correlation and averaging techniques.

It is important to emphasize that the consistency in the circulation of activity demonstrated by these findings in terms of clusters of action potentials generated by groups of neurons, does not indicate that the same consistency can be found in circulation from single neurons to single neurons. As has often been stressed in past studies $[30,33]$, the activity circulates over many coherent pathways, from groups of neurons to groups of neurons, as shown by the fact that correlations are found between clusters of action potentials of different amplitudes recorded by the same microelectrote, between clusters of action potentials recorded by different microelectrodes, or be tween clusters of action potentials and gross waves spontaneous or evoked. Every time a volley of neuronal activity -- spontaneous or evoked -- travels through a network it does not implicate, ilentically, the same neurons that it has implicated in its previous passage or that it will implicate in the next.

For this reason, correlations between the action potentials of single neurons are rare and fleeting. When they are shown in figures such as $4 \mathrm{~B}$ in this report, it is only for the purpose of illustrating the fact that they occasionally can be found. No predictability or causality is suggested or was ever suggested in any study of any correlations between such phenomena, conducted by Verzeano and his collaborators. On the contrary as stated by Verzeano $|26|$ in relation to gross evoked potentials and concomitant neuronal respones: "When a series of such responses containing gross waves and single neuronal discharge are recorded, they show great variability and no conclusions can be drawn about the relations between the gross and neuronal responses... This detailed information on the distribution of neuronal spike amplitudes with respect to time and its relations to the phase of the gross response could not be provided by single neuronal analysis no matter how many (microelectrode) penetrations might be made and no matter how much information might be obtained on the percentage of neurons behaving in one way rather than in some other way." A similar statement was made recently by Ramos et al. [14] who arrived at similar conclusions.

\section{ACKNOWLEDGEMENTS}

The authors are indebted to $\mathrm{F}$. Kuch, J. Otsen, J. Glazer, and $\mathrm{M}$. El Torae for technical assistance.

\section{REFERENCES}

1. Andersen, P. and S. A. Andersson. In: Physiological Basis of the Alpha Rhythm. New York: Appleton-Century-Crofts. 1968.

2. Andersen, P. and J. C. Eccles. Inhibitory phasing of neuronal discharge. Nature (Lond.) 196: 645-647, 1962.
3. Creutzeldt, O. D., S. Watanabe and H. D. Lux. Relations between EEG phenomena and potentials of single cortical cells. 1. Evoked responses atter thalamie and epicortical stimulation. Electroenceph ctin. Netwophysiol 20: $1-18.1966 \mathrm{a}$. 
4. Creutzfeldt, O. D., S. Watanabe and H. D. Lux. Relations between EEG phenomena and potentials of single cortical cells. II. Spontaneous and convulsoid activity. Electroenceph. clin. Neurophysiol. 20: 19-37, 1966b.

5. Dill, R. C., E. Vallecalle and M. Verzeano. Evoked potentials, neuronal activity and stimulus intensity in the visual system. Physiol. Behav. 3: 797-801, 1968.

6. Frigyesi, T. L. Intracellular recordings from neurons in dorsolateral thalamic reticular nucleus during capsular, basal ganglia and midline thalamic stimulation. Brain Res. 48: $157-172,1972$.

7. Grover, F. and J. Buchwald. Correlation of cell size with amplitude of background fast activity in specific brain nuclei. J. Neurophysiol. 33: 160-171, 1970.

8. Kalil, R. and R. Chase. Corticofugal influence on activity of lateral geniculate neurons in the cat. J. Neurophysiol. 33: $459-474,1970$

9. Kusske, J. A. Interactions between thalamus and cortex in experimental epilepsy in the cat. Expl Neurol. 50: 568-578, 1976.

10. Purpura, D. P. Intracellular studies of synaptic organization in the mammalian brain. In: Structure and Function of Synapses, edited by G. D. Pappas and D. P. Purpura. New York: Raven Press, 1972, pp. 257-302.

11. Purpura, D. P. Nature of electrocortical potentials and synaptic organizations in cerebral and cerebellar cortex. Int. Rev. Neurobiol. 1: 47-163, 1959.

12. Purpura, D. P. and B. Cohen. Intracellular recordings from thalamic neurons during recruiting responses. J. Neurophysiol. 25: $621-635,1962$.

13. Purpura, D. P. and R. J. Shofer. Cortical intracellular potentials during augmenting and recruiting responses. I. Effects of injected hyperpolarizing currents on evoked membrane potential changes. $J$. Neurophysiol. 27: 117-132, 1964.

14. Ramos, A, E. Schwartz, and E. R. John. Evoked potential-unit relationships in behaving cats. Brain Res. Bull. 1: 69-75, 1976.

15. Rinaldi, P., M. Sutko, J. H. Mahnke and M. Verzeano. Serotonin in the lateral geniculate. Physiol. Behav. 14: pp. 95-102, 1975.

16. Scheibel, M. E. and A. B. Scheibel. Structural organization of nonspecific thalamic nuclei and their projection toward cortex. Brain Res. 6: 60-94, 1967.

17. Scheibel, M. E., A. B. Scheibel and T. H. Davis. Some substrates for centrifugal control over thalamic cell ensembles. In: Corticothalamic Projections and Somatosensory Activities, edited by T. Frigyesi, E. Rivak and M. D. Yahr. New York: Raven Press, 1972.

18. Sloper, J. J. Dendrodendritic synapses in the primate motor cortex. Brain Res. 34: 186-192, 1971.
19. Teyler, T. J., R. A. Roemer and R. F. Thompson. Relations between gross and unit evoked activity in pericruciate cortex of cat. Physiol. Behav. 6: 375-479, 1971.

20. Velasco, M. and D. B. Lindsley. Role of orbital cortex in regulation of thalamocortical electrical activity. Science 145: $1375-1377,1965$.

21. Velasco, M., J. E. Skinner, K. D. Asaro and D. B. Lindsley. Thalamocortical ablations. Electroenceph. clin. Neurophysiol. 25: 463-470, 1968.

22. Verzeano, $M$. The activity of the neuronal networks in memory consolidation. In: Sleep and Memory, edited by Drucker-Colin and J. McGaugh. New York: Academic Press, in press, 1977.

23. Verzeano, M. The synchronization of brain waves. Acta Neurol. Latinoamer. 9: 297-307, 1963.

24. Verzeano, M. Evoked responses and network dynamics. In: The Neural Control of Behavior, edited by R. E. Whalen, R. F. Thompson, M. Verzeano and N. M. Weinberger. New York: Academic Press, 1970, pp. 27-54.

25. Verzeano, M. Pacemakers, synchronization and epilepsy. In: Synchronization of EEG Activity in Epilepsies, edited by $\mathrm{H}$. Petsche and M. Brazier. Vienna: Springer, 1972.

26. Verzeano, M. The study of neuronal networks in the mammalian brain. In: Bioelectric Recording Techniques, Part A, edited by R. F. Thompson and M. Patterson. New York: Academic Press, 1973.

27. Verzeano, M. Activity of cerebral neurons in the transition from wakefulness to sleep. Science 124: 366-367, 1956.

28. Verzeano, M. and I. Calma. Unit-activity in spindle bursts. $J$. Neurophysiol. 17: 417-428, 1954.

29. Verzeano, M., M. Laufer, P. Spear et S. McDonald. L'activite des reseaux neuroniques dans le thalamus du singe. Actual. Neurophysiol. 6: 223-252, 1965.

30. Verzeano, M., M. Laufer, P. Spear and S. McDonald. The activity of neuronal networks in the thalamus of the monkey. In: Biology of Memory, edited by K. Pribram and D. E. Broadbent. New York: Academic Press, 1970, pp. 239-271.

31. Verzeano, M. and J. H. Mahnke. Serotonin and thalamic synchronization. Physiol. Behav. 9: 649-653, 1972.

32. Verzeano, M. and K. Negishi. Neuronal Activity in Cortical and Thalamic Networks-A study with multiple microelectrodes. $J$. gen. Physiol. 43: 177-195, 1960.

33. Verzeano, M. and K. Negishi. Neuronal activity in wakefulness and in sleep. In: The Nature of Sleep, edited hy G. E. W. Wolstenholme and M. O'Connor. London: Churchill (Ciba Symposium). 1961, pp. 108-130.

34. Wolbarsht, M. L., E. F. MacNichol, Jr. and H. G. Wagner. Glass insulated platinum microelectrodes. Science 132: 1309-1310, 1960. 